\title{
Perforación tras colonoscopia: experiencia en 16 años
}

\author{
M. T. García Martínez, A. Ruano Poblador, L. Galán Raposo, A. M. Gay Fernández y J. R. Casal Núñez \\ Servicio de Cirugía General y Digestiva. Hospital do Meixoeiro. Vigo, Pontevedra
}

\section{RESUMEN}

Objetivo: el propósito de este estudio retrospectivo fue conocer la incidencia de perforaciones de colon por colonoscopia diagnóstica y terapéutica y valorar el manejo de las mismas en nuestro medio.

Pacientes y método: se revisaron las perforaciones por colonoscopias realizadas en nuestro hospital entre enero de 1991 y diciembre de 2006. Se analizaron la finalidad del procedimiento, el mecanismo de la lesión, su presentación clínica y radiológica, el retraso diagnóstico, el estado general de los pacientes, el manejo terapéutico y sus resultados.

Resultados: en el periodo de estudio hubo 15 perforaciones $(0,09 \%)$ de un total de 16.285 colonoscopias realizadas (9 varones, 6 mujeres). Nueve ocurrieron tras colonoscopias diagnósticas y 6 terapéuticas. El 60\% de las perforaciones fueron advertidas por el endoscopista durante el procedimiento (en el 88,6\% de las diagnósticas y en el 16,6\% de las terapéuticas). En el 73,7\% de los casos el dolor con distensión abdominal fue el síntoma más frecuente y en 11 pacientes con pruebas de imagen se apreció gas extraluminal en el $100 \%$ de los casos. El retraso diagnóstico (> 24 horas) ocurrió en el 40\% de los pacientes (rango: 1-6 días). Fueron intervenidos 12 pacientes (80\%) de los que 4 eran ASA II y 8 ASA III/IV. La morbilidad postoperatoria fue del $44,44 \%$ y la mortalidad del 25\%. La evolución de los pacientes tratados conservadoramente fue satisfactoria.

Conclusión: la perforación de colon por colonoscopia es una complicación rara de consecuencias graves e incluso letales. El tratamiento conservador puede realizarse en casos seleccionados y bajo un control clínico estricto. El tipo de cirugía va a depender de la localización, tamaño de la lesión, patología colónica concomitante y grado de contaminación fecal. La mortalidad parece estar más relacionada con el estado general del paciente que con la propia técnica quirúrgica realizada.

Palabras clave: Perforación colon. Colonoscopia. Complicaciones colonoscopia.

\begin{abstract}
Objective: the aim of this retrospective study was to evaluate the incidence of colon perforations from diagnostic and therapeutic colonoscopies, and to assess their management in our hospital.

Patients and method: perforations resulting from colonoscopy in our hospital were reviewed for the period January, 1991 to December, 2006. The study analyzed: purpose of procedure, lesion mechanisms, clinical and radiological presentations, delays in diagnosis, patient status, therapeutic handling, and outcome.

Result: fifteen perforations (0.09\%) (9 males and 6 females) out of a total of 16,285 colonoscopies carried out were seen to have taken place during the study period. Nine of these occurred after diagnostic colonoscopies, and 6 occurred after therapeutic endoscopies. Around $60 \%$ of perforations were detected by the endoscopist while carrying out the procedure $188.6 \%$ during diagnostic endoscopy, and $16.6 \%$ during therapeutic endoscopy). In $73.7 \%$ of cases abdominal pain and distension were the most frequent symptoms; extraluminal gas was seen in $100 \%$ of cases with imaging techniques. Delayed diagnosis ( $>24$ hours) occurred in $40 \%$ of patients (range: 1-6 days). Twelve patients were operated upon (80\%), of whom 4 were ASA II and 8 were ASA III/IV. Postoperative morbidity was $44.44 \%$, and mortality was $25 \%$. The outcome of patients receiving conservative treatment was found to be satisfactory.

Conclusion: perforation of the colon during colonoscopy is a rare complication with serious -even lethal- consequences. Conservative treatment can be provided for selected cases under strict clinical control. Type of surgery will depend on the lesion location and size, concomitant colon pathology, and degree of fecal contamination. Mortality seems to be related to general status rather than the surgical technique used.
\end{abstract}

Key words: Colonic perforation. Colonoscopy. Colonoscopy complications.

García Martínez MT, Ruano Poblador A, Galán Raposo L, Gay Fernández AM, Casal Núñez JE. Perforación tras colonoscopia: experiencia en 16 años. Rev Esp Enferm Dig 2007; 99: 588-592.

Recibido: 30-03-07.

Aceptado: 10-07-07.

Correspondencia: María Teresa García Martínez. Secretaría de Cirugía General. Hospital do Meixoeiro. Crrta do Meixoeiro, s/n. 36200 Vigo, Pontevedra.e-mail: teresa_garcia_martinez@yahoo.es

\section{INTRODUCCIÓN}

La colonoscopia es un procedimiento habitual utilizado en el diagnóstico, tratamiento y seguimiento de las enfermedades del colon. La perforación tras colonoscopia 
es una complicación poco frecuente pero de consecuencias importantes e incluso letales. Su incidencia, en series recientes y de gran volumen ( $>15.000$ colonoscopias) oscila entre el 0,016 y el 0,19\% (1-3). Mientras en algunas series el porcentaje es similar cuando se emplea con fines diagnósticos o terapéuticos (4), en otras es más frecuente como procedimiento terapéutico (5) o significativamente mayor como diagnóstico (6).

Aunque se describen casos específicos tratados conservadoramente e incluso en algunas series se trató de esta manera y con éxito al $100 \%$ de los pacientes (7), en series recientes entre el 33 y el $100 \%$ de los enfermos precisan de una laparatomía urgente tras el diagnóstico $(8,9)$ con un índice de morbilidad entre el 27 y el $39 \%$ y de mortalidad entre el 0 y el $14 \%(4,6,10,11)$.

El propósito del presente estudio fue determinar la incidencia en nuestro hospital de las perforaciones secundarias a colonoscopia y evaluar fundamentalmente su manejo terapéutico y sus resultados.

\section{PACIENTES Y MÉTODO}

Entre enero de 1991 y diciembre de 2006 se realizaron en nuestro hospital 16.285 colonoscopias. Los datos demográficos de los pacientes fueron recogidos del Centro de Codificación de nuestro hospital y se revisaron los informes registrados de las colonoscopias. De los pacientes con diagnóstico de perforación tras colonoscopia se recogieron los siguientes datos: edad, sexo, empleo de sedación y analgesia, puntuación ASA (American Society of Anesthesiology), indicación de la colonoscopia, antecedentes personales, tiempo entre el procedimiento y el diagnóstico de perforación, presentación clínica, hallazgos radiológicos, localización, manejo terapéutico, morbilidad y mortalidad.

\section{RESULTADOS}

En un periodo de 16 años se realizaron 16.285 colonoscopias con un resultado de 15 perforaciones $(0,09 \%)$, el $80 \%$ de las mismas ocurrieron durante la última mitad del periodo de estudio ( 3 perforaciones de 5.638 colonoscopias entre 1991 y 1998 y 12 entre 1999 y 2006). Once se localizaron en sigma, 2 en ciego y 2 en la unión rectosigmoidea. La edad media de los pacientes fue de 70,20 años (rango 33-88 años) y 9 eran varones. En los pacientes con perforación debida a colonoscopia como procedimiento diagnóstico (60\%) la edad media fue de 69,55 años (rango 50-86 años) y como terapéutico de 71,16 años (rango 33-88 años).

Las indicaciones fueron: realización de polipectomía (n: 5), anemia: (n: 4), hemorragia digestiva baja (n: 2), alteración del hábito intestinal (n: 2) argonterapia por angiodisplasia (n: 1) y control por enfermedad de Crohn (n: 1). Dos pacientes habían sido histerectomiza- das y 1 apendicectomizado. Entre las comorbilidades más significativas destacan: tratamiento con anticoagulantes (n: 3), hipertensión portal (n: 2), insuficiencia renal crónica y cardiaca (n: 1), insuficiencia renal crónica (n: 1), enfermedad de Crohn colónica (n: 1), insuficiencia respiratoria crónica y hernia inguinal izquierda (n: 1) y accidente cerebrovascular ( $\mathrm{n}: 1)$.

El 93,33\% de los pacientes con perforación habían sido preparados con lavado anterógrado. El colon no fue preparado en un paciente con accidente cerebrovascular y en 2 se informó como mal preparado. En 10 casos $(66,66 \%)$ se realizó el procedimiento bajo sedación y analgesia (midazolam y miperidina) y de ellos la lesión fue objetivada por el endoscopista en el $60 \%$ de los casos, índice similar al de los casos en los que se realizó la colonoscopia sin medicación alguna.

Se informó de la dificultad de progresión del colonoscopio por la existencia de un sigma redundante con presencia de divertículos en 5 pacientes y por sigma adherido a un saco herniario de hernia inguinal izquierda en un caso. La lesión fue advertida por el endoscopista en el $88,6 \%$ de las colonoscopias diagnósticas y en el 16,6\% de las terapéuticas, 3 consultaron transcurridas 24 horas desde el procedimiento, 1 tras 48 horas y 2 a los 4 y 6 días respectivamente. La causa de la perforación fue identificada como mecánica en 12 pacientes (por traumatismo directo de la punta del colonoscopio en 7, secundaria a polipectomía en 5 y por tratamiento con argonterapia en 1) y neumática en 3 pacientes.

Tan sólo 1 paciente cursó sin sintomatología alguna y fue diagnosticado a los 4 días de realizada la colonoscopia por presentar en el líquido de diálisis peritoneal contenido fecaloideo. Los síntomas más frecuentes fueron dolor y distensión abdominal $(73,73 \%)$, sólo distensión abdominal $(6,66 \%)$ o dolor $(13,33 \%)$. Un paciente cuya perforación fue objetivada transcurridas 24 horas cursó con un cuadro de enfisema subcutáneo sin otra sintomatología acompañante. De los 11 pacientes a los que se realizaron pruebas de imagen (radiología simple o tomografía axial computarizada abdominal) estas fueron positivas a neumoperitoneo en todos casos de los cuales en uno se acompañaba de neumorretroperitoneal y mediastínico (Fig. 1).

De los 12 pacientes intervenidos quirúrgicamente 4 eran ASA II, 6 ASA III y 2 ASA IV. En 8 casos la cirugía se realizó de manera inmediata (menos de 3 horas de la realización de la colonoscopia), 2 transcurridas 24 horas, 1 tras 48 horas y 1 a los 4 días. Se realizó una intervención de Hartmann en 4 pacientes, sutura primaria en 4 (una de ellas por vía laparoscópica), colectomía derecha y anastomosis ileocólica en 2, sigmodectomía y anastomosis primaria en 1 y colectomía subtotal con anastomosis íleo-rectal en 1 paciente con angiodisplasia de colon derecho, diverticulosis de colon izquierdo y perforación de $12 \mathrm{~cm}$ localizada en sigma. Fallecieron en el postoperatorio 3 pacientes $(25 \%), 2$ de ellos habían sido intervenidos de manera inmediata y 1 a las 24 horas. Las causas de fallecimiento fueron shock séptico de origen 


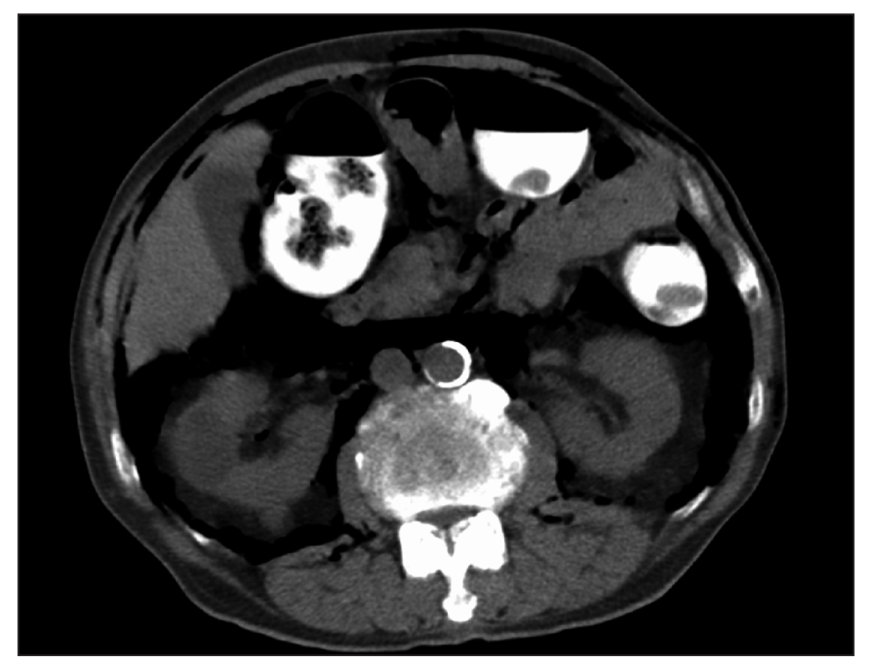

Fig. 1. Neumoperitoneo, retroneumoperitoneo y enfisema subcutáneo.

abdominal en un paciente ASA III de 86 años de edad y hemorragia digestiva alta en 2 pacientes, con antecedentes de hipertensión portal, de 82 y 86 años y ASA III y IV respectivamente (Tabla I).

La morbilidad postquirúrgica fue del 44,44\%: neumonía (n: 2), absceso subfrénico, drenado percutáneamente (n: 1) e infección de la herida quirúrgica (n: 1). La estancia media postoperatoria fue de 16 días (rango: 7-43 días).

Un paciente presentó dolor en fosa iliaca izquierda tras la colonoscopia y fue diagnosticado radiológicamente a los 6 días de perforación de sigma y tratado médicamente con resultado satisfactorio. No hubo complicaciones en los 3 pacientes que fueron tratados conservadoramente.

Tabla I. Perforación por colonoscopia. Tratamiento quirúrgico

\begin{tabular}{lcccccc}
\hline Caso & Sexo & Edad & ASA & Evolución & Cirugía & Mortalidad \\
\hline 1 & V & 50 & IV & 4 días & Hartmann & No \\
2 & H & 86 & III & Inmediato & Resección y anastomosis & Sí \\
3 & H & 81 & III & Inmediato & Sutura & No \\
4 & V & 82 & IV & Inmediato & Sutura & Sí \\
5 & V & 67 & II & Inmediato & Hartmann & No \\
6 & V & 86 & III & 24 horas & Colectomía derecha & Sí \\
7 & V & 33 & II & 48 horas & Hartmann & No \\
8 & V & 85 & II & Inmediato & Sutura & No \\
9 & V & 45 & III & Inmediato & Colectomía y & No \\
& & & & & anastomosis ileorrectal & \\
10 & H & 59 & III & Inmediato & Sutura & No \\
11 & H & 76 & II & Inmediato & Hartmann & No \\
12 & V & 81 & III & 24 horas & Colectomía derecha & No \\
\hline
\end{tabular}

\section{DISCUSIÓN}

Para Wexner y cols. (12) el índice de complicaciones no está asociado al nivel de experiencia ni al número de colonoscopias realizadas anualmente. En nuestra serie la incidencia de perforación fue del 0,092\%. En la primera mitad de nuestro estudio se realizaron un total de 5.638 colonoscopias con un resultado de 3 perforaciones
$(0,05 \%)$ siendo en la segunda mitad del $0,11 \%$ en un volumen de 10.647 colonoscopias por lo que no hemos encontrado diferencias entre ambos grupos. En este segundo periodo las perforaciones ocurrieron en colonoscopias realizadas por 4 endoscopistas con más de 10 años de experiencia y más de 400 colonoscopias realizadas cada uno. Pucher y cols. (13) comunican que la frecuencia de complicaciones es proporcional al nivel de experiencia, sin embargo esto no ocurrió en nuestra serie por lo que creemos que los factores de riesgo comunicados por varios autores $(2,4,10,14,15)$, como la existencia de enfermedad inflamatoria, antecedentes de radiación pélvica, presencia de divertículos y cirugía abdominal previa juegan un papel fundamental en el incremento del riesgo de perforación. La existencia de estenosis de colon por enfermedad de Crohn, cirugía abdominal previa y diverticulosis sigmoidea con el sigma alojado en un saco herniario inguinal en 1 paciente, fueron, a nuestro criterio, posibles factores de riesgo que estaban presentes en 1,3 y 5 pacientes respectivamente.

Mientras en algunas series el índice de perforaciones por colonoscopia diagnóstica se sitúa entre el 42 y el $50 \%$ $(2,4,8,14,16)$ en otras oscila entre el 63 y el $72 \%(5,6,17,18)$. La causa de estas perforaciones puede ser de origen mecánico o por barotrauma. La lesión por la punta del colonoscopio en la pared del colon por maniobras inadecuadas, la creación de un asa en alpha o la penetración del endoscopio a través de la mucosa y su avance sin visualizar la luz son las causas más frecuentes de perforación colónica (19-21). El error en diferenciar la luz de un divertículo de la luz del colon, la presencia de adherencias por cirugía previa, la existencia de un sigma redundante y la mayor incidencia de divertículos en la región sigmoidea hacen que este segmento sea más propenso a ser lesionado $(4,6,17,20,21)$. La perforación por barotrauma, es decir, como resultado de un insuflación excesiva de gas, provoca con mayor frecuencia perforación a nivel cecal $(4,22)$. Aunque también puede ocurrir en el colon sigmoideo debido a que las flexuras del colon pueden posibilitar la creación de un segmento en el cual se genere una alta presión durante la insuflación de gas (23). Esto pudo haber ocurrido en nuestros dos casos de perforación atribuibles a barotrauma en uno de los cuales el sigma estaba acodado y adherido al saco de una hernia inguinal. En nuestra serie la mayoría de los casos de perforación durante la colonoscopia diagnóstica fueron debidos a traumatismo directo de la pared del colon por la punta del colonoscopio, localizándose a nivel de la unión rectosigmoidea y en el sigma, en 1 y 6 pacientes respectivamente, hallándose la lesión localizada en todos los casos en el borde antimesentérico y de un tamaño que osciló entre los 1,5 y $12 \mathrm{~cm}$ longitudinalmente.

Las perforaciones durante la colonoscopia terapéutica pueden ocurrir por mecanismos similares a la diagnóstica, pero con mayor frecuencia son causadas tras biopsias, polipectomías, electrocauterio o empleo de argonterapia como tratamiento de malformaciones arteriovenosas 
$(2,3,11,18,24)$. Este tipo de perforaciones en nuestra experiencia y en la de otros autores son de un tamaño considerablemente menor a las provocadas por la punta del colonoscopio y su reconocimiento suele ser tardío $(2,9)$.

El dolor y la distensión abdominal es la presentación más frecuente $(10,18)$, no obstante en algunos casos la sintomatología está ausente o se inicia pasados días e incluso semanas $(4,7,11,17)$. En este estudio el retraso diagnóstico fue del 40\%, similar a otras series $(4,7)$ con una media de 2,5 días (rango: 1-6) y en el $83 \%$ de los casos se había realizado la colonoscopia con fines terapéuticos por lo que creemos que las perforaciones han ocurrido como resultado del contacto prolongado del láser en el tratamiento de una angiodisplasia (24) y por lesión térmica transmural en el transcurso de la aplicación de electrocoagulación tras polipectomía $(25,26)$.

El uso de anestésicos no incrementa el riesgo de perforación (27) ni se acompaña de complicaciones graves (28) y nosotros no hemos encontrado diferencias en el retraso diagnóstico con la utilización de fármacos sedantes y analgésicos durante el procedimiento, sin embargo el grado de sedación y analgesia es controvertido y así mientras para algunos autores (29) la sedación profunda es un factor importante en la valoración de signos y síntomas, otros la utilizan en la mayoría de sus pacientes sin comunicar efectos adversos (28).

Los hallazgos de la radiología simple pueden mostrar gas a nivel pericólico, libre en la cavidad abdominal o extensión del mismo en el espacio retroperitoneal $(17,18,30)$. En las perforaciones rectales o a nivel rectosigmoideo el gas puede extenderse a lo largo del músculo psoas, mediastino y tejidos subcutáneos $(7,30,31)$. Si la radiología simple no sugiere perforación una tomografía puede ayudar al diagnóstico descubriendo gas libre, microperforaciones o abscesos (11).

La ausencia de gas extracolónico no excluye el diagnóstico de perforación (17) y la sola presencia del mismo no es suficiente justificación para realizar cirugía $(11,16,25$, 31,32). El manejo terapéutico de estos pacientes es controvertido y el fracaso del tratamiento conservador que obliga a una posterior laparotomía es en algunas series igual o superior al $50 \%$ de los casos $(2,15)$. Algunos autores $(33,34)$ defienden la cirugía inmediata basándose en que el fracaso del tratamiento conservador incrementa la contaminación y la inflamación aumentando la morbilidad y la mortalidad postoperatoria. Las perforaciones que resultan de una colonoscopia terapéutica suelen ser de pequeño tamaño y la contaminación abdominal mínima. $\mathrm{Si}$ a esto añadimos una preparación adecuada del colon y las condiciones generales del paciente son buenas, posiblemente este tipo de perforaciones respondan a menudo de forma satisfactoria a tratamiento conservador $(7,8,26,29)$. A los pacientes de nuestra serie que fueron tratados conservadoramente se les había realizado polipectomía y presentaban signos radiológicos de gas extraluminal y un absceso pericólico en uno de ellos por lo que pensamos que la cirugía inmediata debe reservarse para aquellos pacientes cuya perforación es diagnosticada por el endoscopista, generalmente durante el procedimiento diagnóstico (2) y que no puedan ser manejadas terapéuticamente por este (35) y cuando los signos y síntomas sean indicativos de afectación peritoneal, por lo que el éxito del tratamiento conservador va a depender de la estabilidad clínica del paciente y de la evolución de su sintomatología $(15,25)$ por lo que se recomienda en estos casos una observación clínica estricta $(11,18)$.

El tipo de cirugía dependerá del tamaño de la lesión, grado de contaminación, patología colónica asociada y estado general del paciente $(8,10,11)$ por lo que se incluyen dentro de las opciones quirúrgicas la sutura de la perforación, resección y anastomosis primaria con o sin estoma de protección y resección intestinal y estoma en aquellos pacientes con una contaminación fecal extensa, múltiples comorbilidades o inestabilidad hemodinámica $(2,4,16-18,32)$.

El índice de mortalidad postoperatoria, en la literatura consultada, oscila entre el 0 y el $50 \%(4,10,11,16,17,26,34)$. Fallecieron en la presente serie 3 pacientes (25\%), 1 ASA IV y 2 ASA III, a los cuales se les había realizado sutura, resección y anastomosis primaria y colectomía derecha respectivamente tras un diagnóstico inmediato de la perforación en 2 de ellos y un retraso de 24 horas tras la colonoscopia en 1. Por ello opinamos que la mortalidad depende más de las condiciones generales del paciente, en estos casos, que del tipo de cirugía realizada (4).

La vía laparoscópica constituye una alternativa segura y efectiva en el tratamiento de esta entidad (36-39). A un paciente de nuestra serie se le practicó sutura de la perforación por vía laparoscópica y su estancia en el hospital fue de 9 días, similar a la de los 2 pacientes en los que se realizó la misma técnica por vía abierta. Una mayor experiencia y documentación sobre la utilización de la laparoscopia en el tratamiento de las perforaciones por colonoscopia podría aportarnos datos sobre una posible reducción en la morbilidad y en la estancia hospitalaria de estos pacientes.

\section{CONCLUSIÓN}

Aunque las perforaciones por colonoscopia son infrecuentes, la morbilidad y mortalidad postoperatorias pueden ser significativas. La cirugía inmediata se realiza en la mayoría de los casos en los que la lesión es advertida por el endoscopista durante el procedimiento con fines diagnósticos. Cuando la perforación es debida a una colonoscopia terapéutica, especialmente tras polipectomía, los pacientes pueden ser tratados generalmente de forma conservadora bajo una vigilancia clínica estricta. El tipo de cirugía va a depender de la localización, tamaño de la lesión y patología colónica concomitante y podrá realizarse en un solo tiempo salvo en aquellos casos en los que exista una contaminación fecal extensa. La mortalidad parece estar más relacionada con el estado general del paciente que con la propia técnica quirúrgica. 


\section{BIBLIOGRAFÍA}

1. Rathgaber SW, Wick TM. Colonoscopy completion and complication rates in a communnity gastroenterology practice. Gastrointestinal Endoscopy 2006; 64: 556-62.

2. Coob WS, Heniford T, Sigmon LB, Hasan R, Simms C, Kercher KW, et al. Colonoscopic perforations: Incidence, management and outcomes. Am Surg 2004; 70: 750-7.

3. Anderson ML, Pasha TM, Leighton JA. Endoscopic perforation of the colon: Lessons from a 10 year study. Am J. Gastroenterol 2000; 95: 3418-22.

4. Orsoni P, Berdah S, Verrier C, Caamano A, Sastre B, Boutboul R, et al. Colonic perforation due to colonoscopy: A retrospective study of 48 cases. Endoscopic 1997; 29: 160-4.

5. Ettersperger L, Zeitoun P, Thiefin G. Colonic perforations complicating colonoscopic. A propos of 15 consecutive cases observed over 16 years. Gastroenterol Clin Biol 1995; 19: 1018-22.

6. Korman LY,Overholf BF, Box T, Winker CK. Perforation during colonoscopic in endoscopic ambulatory surgical centers. Gastroint Endosc 2003; 58: 554-7.

7. Ker TS, Wasserberger N, Beart RW. Colonoscopic perforation and bleeding of the colon can be treated safely without surgery. Am Surg 2004; 70: 922-4.

8. Lo AY, Beaton HL. Selective management of colonoscopic perforations. J Am Coll Surg 1994; 179: 333-7.

9. Dafnis G, Ekbom A, Pahlman L, Blomquist P. Complications of diagnostic and therapeutic colonoscopy within a defined population in Sweden. Gastrointest Endosc 2001; 54: 302-9.

10. Farley DR, Bannon MP, Zietlow SP, Pemberton JH, Ilstrup DM, Larson DR. Management of colonoscopic perforations. Mayo Clin Proct 1997; 72: 729-33.

11. Iqbal CW, Chun YS, Farley DR. Colonoscopic perforations: A retrospective review. J Gastrointest Surg 2005; 9: 1229-35.

12. Wexner SD, Garbus JE, Sing JJ, SAGES Coloscopic Study Outcomes Group. A prospective analysis of 13580 colonoscopies. Reevaluation of credentialing guidelines. Surg Endosc 2001; 15: 251-61.

13. Puchner R, Allinger S, Doblhofer F, Wallner M, Knoflach P. Complications of diagnostic and interventional colonoscopy. Wien Klin Wochenschr 1996; 108: 142-6.

14. Araghizadeh FY, Timmcke AE, Opelka FG, Hicks TC, Beck DE. Colonoscopic perforations. Dis Colon Rectum 2001: 44: 713-6.

15. Gedebou TM, Wong RA, Rappaport WD, Jaffe P, Kahsai D, Hunter GC. Clinical presentation and management of iatrogenic colon perforations. Am J Surg 1996; 172: 454-8.

16. Carpio G, Albu E, Gumbs MA, Gerst PH. Management of colonic perforation after colonoscopy. Report of three cases. Dis Colon Rectum 1989; 32: 624-6.

17. Garbay JR, Suc B, Rotman N, Fourtanier G, Escat J. Multicentre study of surgical complications of colonoscopy. Br J Surg 1996; 83: 42-4.

18. Tulchinsky H, Madhala-Givon O, Wasserberg N, Lelcuk S, Niv Y. Incidence and management of colonoscopic perforations: 8 years'ex- perience. World J Gastroenterol 2006; 12: 4211-3.

19. Damore LJ, Rantis PC, Vernava AM, Longo WE. Colonoscopic perforations. Etiology, diagnosis and management. Dis Colon Rectum 1996; 39: 1308-14.

20. Williams C, Teague R. Colonoscopy. Gut 1973; 14: 990-1003.

21. Livstone EM, Kerstein MD. Serosal tears following colonoscopic. Arch Surg 1976; 111: 88.

22. Picciano LD, Hansel BC, Luchette FA. Insufflation: Complications and recommendations. Surg Endosc 1995; 9: 834-6.

23. Brayco CM, Kozarek RA, Sanowski RA, Howells T. Diverticular rupture during colonoscopic. Fact or fancy? Dig Dis Sci 1984; 29: 427-31.

24. Ghazi A, Grossman M. Complications of colonoscopy and polipectomy. Surg Clin North Am 1982; 62: 889-96.

25. Christie JP, Marrazzo J. Mini-perforation of the colon-not all postpolypectomy perforations require laparotomy. Dis Colon Rectum 1991; 34: 132-5.

26. Hall C, Dorricott NJ, Donovan IA, Neoptolemos JP. Colon perforatiopn during colonoscopic: Surgical versus conservative management. Br J Surg 1991; 78: 542-4.

27. Martínez J, Casellas JA, Aparicio JR, Garmendia M, Amorós A. Seguridad de la administración de propofol por el personal de la unidad de endoscopia digestiva. Gastroenterol Hepatol 2007; 30: 105-9.

28. Kjaergard H, Nordkild P, Geerdsen J, Dyrberg V. Anaesthesia for colonoscopy. An examination of the anaesthesia as an element of risk at colonoscopy. Acta Anaesthesiol Scand 1986; 30: 60-3.

29. Kavin H, Sinecrope F, Esker AH. Magement of perforation of the colon at colonoscopy. Am J Gastroenterol 1992; 87: 161-7.

30. Humphreys F, Hewetson KA, Dellipiani AW. Massive subcutaneous emphysema following colonoscopy. Endoscopy 1984; 16: 160-1.

31. Nivatvongs S. Complications in colonoscopic polypectomy: And experience with 1555 polypectomies. Dis Colon Rectum 1998; 29: 825-30.

32. Vincent M, Smith LE. Management of perforation due colonoscopy. Dis Colon Rectum 1983; 26: 61-3.

33. Soon JC, Shang NS, Goh PM, Rauff A. Perforation of the large bowel during colonoscopic in Singapure. Am Surg 1990; 56: 215-8.

34. Barbagallo F, Castello G, Latteri S, Grasso E, Gagliardo S, La Greca $\mathrm{G}$, et al. Successful endoscopic repair of an unusual colonic perforation following polypectomy using endoclip device. World J Gastroenterol 2007; 13: 2889-91.

35. Friedrichs O. Diagnose und therapie der endoskopischen dickdermperforation. Med Welt 1983; 34: 75-6.

36. Miyahara M, Kihno S, Shimoda K, Bandoh T, Chikuba K, Maeo S, et al. Laparoscopic repair of a colonic perforation sustained during colonoscopy. Surg Endosc 1996; 10: 352-3.

37. Velez MA, Riff DS, Mule JM. Laparoscopic repar of a colonoscopic perforation. Surg Endosc 1997; 11: 387-9.

38. Wullstein Ch, Köppen MO, Gross E. Laparoscopic treatment of colonic perforations related to colonoscopy. Surg Endosc 1999; 13: 484-7.

39. Agresta F, Michelet I, Mainente P, Bedin N. Laparoscopic management of colonoscopic perforations. Surg Endosc 2000; 14: 592-3. 